\title{
The value of complete remission according to positron emission tomography prior to autologous stem cell transplantation in lymphoma: a population-based study showing improved outcome
}

Kristina Noring

Karolinska Institutet

Mattias Carlsten

Karolinska Institutet

Kristina Sonnevi

Karolinska Institutet

Björn Wahlin ( $\nabla$ bjom.wahlin@gmail.com )

Karolinska Institutet https://orcid.org/0000-0003-3566-8847

Research article

Keywords: lymphoma, B cell, T cell, ASCT, autologous stem-cell transplantation, PET/CT, positron emission tomography/computerized tomography, PET

Posted Date: December 31st, 2020

DOI: https://doi.org/10.21203/rs.3.rs-136865/v1

License: () (1) This work is licensed under a Creative Commons Attribution 4.0 International License. Read Full License 


\section{Abstract \\ Background}

Chimeric antigen-receptor T-cell and bispecific antibody therapies will likely necessitate a reconsideration of the role of autologous stem-cell transplantation (ASCT) in lymphoma. Patients who are likely to profit from ASCT need to be better identified.

\section{Methods}

Here, we investigated the value of positron emission tomography/computerized tomography (PET/CT) before ASCT. All 521 patients transplanted for lymphoma 1994-2019 at Karolinska (497 conditioned with BEAM) were included.

\section{Results}

Outcome improved over three calendar periods 1994-2004, 2005-2014, 2015-2019 (2-year overall survival [0S]: 66\%, 73\%, 83\%; P = 0.018). Non-relapse mortality (NRM) at 100 days over the three periods were $9.8 \%, 3.9 \%, 2.9 \%$, respectively. The OS improvement between 1994-2004 and 2005-2014 was due to lower NRM $(P=0.027)$, but the large OS advance from 2015 was not accompanied by a significant reduction in NRM $(P=0.6)$. The fraction of PET/CT as preASCT assessment also increased over time: 1994-2004, 2\%; 2005-2014, 24\%; 2015-2019, 60\% ( $\mathrm{P}<0.00005)$. Complete responses (PET/CT-CR) were observed in $77 \%$ and metabolically active partial responses (PET/CT-PR) in $23 \%$. PET/CT-CR was a predictor for survival in the entire population (P $=0.0003$ ), also in the subpopulations of aggressive B-cell $(P=0.004)$ and peripheral T-cell $(P=0.024)$ lymphomas. Two-year OS and progression-free survival $(\mathrm{OS} / \mathrm{PFS})$ for patients in PET/CT-CR were in relapsed/refractory aggressive B-cell lymphoma 87\%/75\% and peripheral T-cell lymphoma $91 \% / 78 \%$. The corresponding figures in PET/CT-PR were $43 \% / 44 \%$ and 33\%/33\%. Patients with solitary PET/CT-positive lesions showed acceptable outcome with ASCT followed by local irradiation (2-year OS/PFS 80\%/60\%). CT was less discriminative: 2-year OS/PFS: CT-CR, 76\%/66\%; CT-PR, 62\%/51\%. Outcome was inferior after BEAC compared with BEAM conditioning.

\section{Conclusions}

We conclude that the improved outcome reflects better, PET/CT-informed, identification of patients who should proceed to ASCT. The excellent survival of patients in PET/CT-CR indicates that ASCT should remain part of standard therapy for lymphoma.

\section{Introduction}

Even though effective non-chemotherapeutic treatment options for lymphomas, such as chimeric antigen-receptor (CAR)-T cell and bispecific antibody therapies, are rapidly emerging, high-dose chemotherapy followed by autologous stem-cell transplantation (ASCT) remains the standard therapeutic option for many patients. Infections are the most common complication after ASCT and the largest contributor to non-relapse mortality (NRM) [1]. Another concern is the potential risk of developing secondary myeloid malignancies [1]. Careful patient selection prior to ASCT is essential to maximize patient benefit while keeping the rate of complications as low as possible. Additional tools are needed to further improve patient selection.

We have previously investigated stem-cell harvest yields and clinical characteristics which affect NRM, other toxicities, and the development of secondary myeloid disease, to help inform on timing and patient selection [2]. However, another important aspect of patient selection is the strength of the indication, which differ by diagnosis, primary or relapsed/refractory setting, and remission status.

Historically, computerized tomography (CT) has been used to assess patients' remission status prior to ASCT. The combination of 18-fluorodeoxyglucose positron emission tomography and CT (PET/CT) enables radiologic mapping of metabolic activity and can be used for identifying and staging the lymphoid malignancies which have elevated glucose consumption. PET/CT is superior to $\mathrm{CT}$ in discriminating between malignant and non-malignant residual tissue, decreasing the risk of incorrectly identifying radiologic remnants post-chemotherapy as active lymphoma [3]. The utility of PET/CT before ASCT to predict outcome has been shown in several lymphoma entities [4-16], although negative series also exist [17, 18]. In these studies, several different conditioning regimens were used, and the timing of the PET/CT differed. We wanted to investigate whether outcome after ASCT had improved with the increasing use of pre-transplant PET/CT and to assess outcome in 521 consecutive lymphoma patients treated with ASCT (497 conditioned with BEAM) in a population-based single-center study.

\section{Material And Methods}

All lymphoma patients who underwent ASCT at the Haematology Unit, Karolinska University Hospital, from 1 January 1994 until 31 October 2019 were included in this retrospective single-center observational study (excluding patients with primary CNS lymphoma). The material is population-based, since the Haematology Unit is the only site conducting this procedure in the Stockholm-Gotland Healthcare Region (population, 2.4 million). Patient data were extracted from electronic medical files. BEAM was dosed day - 7: BCNU $300 \mathrm{mg} / \mathrm{m}^{2}$ 4-hour infusion, intrathecal methotrexate $12 \mathrm{mg}$; days - 6, -5, -4, -3: etoposide $200 \mathrm{mg} / \mathrm{m}^{2}$ 2-hour infusion, cytarabine $400 \mathrm{mg} / \mathrm{m}^{2}$ 12-hour infusion (day - 3 also intrathecal methotrexate $12 \mathrm{mg}$ ); day - 2: melphalan $140 \mathrm{mg} / \mathrm{m}^{2}$ (intrathecal methotrexate was omitted in patients with indolent, T-cell and Hodgkin lymphoma). BEAC was dosed day - 6: BCNU $300 \mathrm{mg} / \mathrm{m}^{2} \mathrm{4}$-hour infusion; days $-5,-4,-3,-2$ : etoposide $100 \mathrm{mg} / \mathrm{m}^{2}$ twice daily, cytarabine $100 \mathrm{mg} / \mathrm{m}^{2}$ 0.5-hour infusion twice daily, cyclophosphamide $35 \mathrm{mg} / \mathrm{kg}$ once and mesna 
$14 \mathrm{mg} / \mathrm{kg}$ four times daily. BCNU-thiotepa was dosed BCNU $400 \mathrm{mg} / \mathrm{m}^{2}$ 1-hour infusion day - 6; thiotepa $5 \mathrm{mg} / \mathrm{kg}$ twice daily days - 5 and - 4 . Investigations of stem-cell harvests (median, 6 million CD34 + cells per $\mathrm{kg}$ ), NRM, and procedure-related toxicities have been published before [2]. Refractory disease was defined as a previous failure of chemotherapy or relapse within 3 months of treatment.

\section{CT and PET/CT}

Complete and partial response (CR; PR) were defined as responses with at least $50 \%$ reduction of lymphoma size and separated by the absence or presence of a lymph node or focal lesion in any organ $\geq 1$ centimetre, or as specified by the reviewing radiologist. PET/CT responses were defined according to the Lugano classification (PET/CT-CR was Deauville score $\leq 3$ ) [3]. PET/CT-PR denotes a shrinking of lymphoma mass but still metabolically active disease.

\section{Statistical analysis}

Patients were followed from ASCT until death or last follow-up (November 2019). Depending on the nature of the independent variables, relationships between them were investigated using the $\chi^{2}$, Wilcoxon or Spearman test. Overall, lymphoma-specific, and progression-free survivals (OS; LSS; PFS) were calculated from the day of ASCT until the day of death (OS), death from lymphoma (LSS), death or progression of disease (PFS). Univariate and multivariate analyses were conducted using Kaplan-Meier curves and Cox regression; the proportional hazards assumption was checked with graphs based on Schoenfeld residuals. All P values are two-tailed and calculated using Stata 14.2 (StataCorp, College Station, TX, USA). P<0.05 was considered significant.

This study was approved by the Ethics Committee, Stockholm (Ref no. 2012/783 - 31/3 with Amendments 2015/327 - 32 and 2016/2379-32).

\section{Results}

521 lymphoma patients underwent ASCT between 1994 and 2019 (Table 1). Most patients were male (63\%) and the median age was 57 years (range, 18-72). At last follow-up (November 2019), the median follow-up time in survivors was 5.3 years (range, 0.1-24.3). The median OS, LSS, and PFS were 13.2, not reached, and 5.7 years (Fig. 1A). OS and PFS at 5 years were $66 \%$ and $52 \%$ (Table 2). The conditioning regimen was BEAM in 497 patients. Because of occasional melphalan shortages from 2015 onwards, BEAC was used in 20 patients. Three patients with CNS relapse of systemic lymphoma were conditioned with BCNU-thiotepa and the first patient in 1994 received cyclophosphamide-total body irradiation. There was improved outcome over the three calendar periods 1994-2004, 2005-2014, 2015-2019 (2-year OS: 66\%, 73\%, 83\%; P = 0.018; Fig. 1B), partly explained by decreasing NRM (at 100 days, $9.8 \%$, 3.9\%, $2.9 \%$, respectively; previously thoroughly described [2]). The OS improvement between 1994-2004 and 2005-2014 was due to lower NRM (P=0.027), but the 10-percentage point OS advance between 2005-2014 and 2015-2019 ( $P=0.029)$ was not accompanied by a significant reduction in NRM $(P=0.6)$. Rituximab use increased in B-cell disease $(30 \%, 96 \%, 100 \%$ over the three calendar periods; $\mathrm{P}<0.00005)$, but could not explain the better outcome (Table 1$)$. Neither was it caused by changes in conditioning, because the BEAM regimen never changed, and in the last calendar period, there was a clear tendency for inferior outcome in patients treated with BEAC compared with BEAM (Fig. 1C), with $P=0.059$ for OS, $P=0.034$ for LSS, and P $=0.062$ for PFS. When BEAC patients were excluded, not only OS and NRM, but also LSS was significantly better in the last calendar period $(P=0.040)$, suggesting the emergence of a new lymphoma-specific survival factor.

\section{The emergence of PET/CT}

We compared the prognostic value of pre-transplant CT and PET/CT. Cases were excluded if the last radiology evaluation prior to ASCT was PR but conducted interim (before the last course of chemotherapy prior to conditioning). All assessments showing CR in the last evaluation were included, either when conducted interim or directly before the ASCT. Thus, the last evaluation of response prior to ASCT was CT in 235 patients (62\%) and PET/CT in 136 (36\%), the remaining 8 (2\%) were assessments using MRI, bone marrow biopsy, or palpation. Over time, PET/CT became increasingly common. PET/CT constituted $2 \%$ and CT $98 \%$ of the final evaluations $1994-2004$, the corresponding numbers were $24 \%$ and $75 \% 2005-2014$, and $60 \%$ and $37 \% 2015-2019$ ( $P<0.0005$ ). Of patients evaluated with PET/CT prior to ASCT, $77 \%$ were in CR and $23 \%$ in PR (Table 3).

Both with CT and PET/CT, PR compared with CR was a poor prognostic marker (OS, $\mathrm{P}=0.035, \mathrm{P}=0.0003$, respectively). However, $\mathrm{PET} / \mathrm{CT}$ was markedly more powerful than CT (Table 3; Fig. 1D). For example, at 2 years, the OS rates were in CT-CR 76\% and CT-PR 62\%, and in PET/CT-CR 93\% and PET/CT-PR 55\% (Table 3). Because CT had been conducted more in the earlier times with higher NRM, the analysis was repeated with restriction to the last calendar period (2015-2019) with similar results (2-year OS: CT-CR 81\%, CT-PR 70\%, PET/CT-CR 96\%, PET/CT-PR 63\%). At the start of conditioning, in patients with PET/CT$\mathrm{CR}, 68 \%$ had normal C-reactive protein ( $<3 \mathrm{mg} / \mathrm{L}$ ), 25\% 3-10 mg/L, and $7 \% \geq 10 \mathrm{mg} / \mathrm{L}$, whereas in PET/CT-PR, the corresponding numbers were $41 \%$, 38\%, and $21 \%(P=0.005)$. There were no $C$-reactive protein differences between CT-CR and CT-PR $(P=0.65)$.

We further investigated the patients transplanted in PR: 19 were given a planned local therapy after ASCT (18, irradiation; 1, splenectomy). That approach appeared useful in the 10 patients where the local therapy was PET-guided (2-year OS/PFS 80\%/60\%) but not in those 9 where CT defined PR (2-year OS/PFS 44\%/44\%; Fig. 1E). The PET/CT-PR patients given local therapy (PET/CT-PR-local) was an intermediate prognostic group between PET/CT-CR and PET/CT-PRgeneral (several FDG-avid sites; Table 3). With respect to OS and PFS, PET/CT-PR compared with PET/CT-CR showed inferior outcome with hazard ratios (HR) 4.0 (95\% confidence interval [Cl], 1.8-9.1) and 2.4 (95\% Cl, 1.3-4.6). For comparison, CT-PR compared with CT-CR had HR 1.5 (95\% Cl, 1.0-2.2) for OS and HR $1.6(95 \% \mathrm{Cl}, 1.2-2.3)$ for PFS.

\section{Primary and transformed aggressive B-cell lymphomas}

In total, 228 (121 primary and 107 transformed) patients were transplanted for aggressive B-cell lymphoma. Outcome did not differ between diffuse large Bcell or other types of primary aggressive lymphoma; likewise, there were no differences between transformed follicular or other transformed indolent lymphomas, nor, overall, between primary or transformed aggressive lymphomas (Table 2), and the curves were roughly similar (Fig. 2A-B). There were no 
outcome differences between primary or transformed aggressive lymphoma in the upfront or relapsed/refractory setting (Fig. 2C), why the 228 aggressive Bcell patients were subsequently grouped for statistical power. Patients transplanted upfront in first remission had better outcome than those who had had relapsed or refractory disease $(O S, P=0.003 ; L S S, P=0.010 ; P F S, P=0.031 ;$ Fig. $2 D)$, similarly, the number of prior lines of chemotherapy was highly predictive for outcome (OS, $P=0.001$; LSS, $P=0.0002 ; P F S, P=0.0002$; Fig. $2 E)$. $P E T / C T$ was a valuable predictive tool for $O S(P=0.004)$, LSS $(P=0.006)$, and $P F S(P=$ 0.032 ), while remission status by CT had no value (Fig. 2F; Table 3 ).

\section{Indolent B-cell lymphomas}

Short- and long-term outcome of the 49 patients with indolent lymphoma are shown in Table 2; late relapses were seen. In follicular lymphoma, $31 \%$ were alive and in remission after 10 years. Outcome was almost identical (Fig. 3A; Table 2) in transformed and not-transformed indolent lymphoma, apart from an expected initial higher mortality in transformed disease. There were too few events for investigating the PET/CT method in not-transformed indolent lymphoma. When combining transformed and not-transformed disease, PET/CT was highly predictive for OS $(P=0.0003)$ and PFS $(P=0.013)$, while $C T$ was not predictive ( $P>0.5$ for both comparisons).

\section{Mantle cell lymphoma}

85 out of 99 mantle cell lymphoma patients were transplanted upfront in or according to the Nordic MCL2 trial [19]. These patients showed excellent outcome with median PFS 9.4 years (Fig. 3B). Patients with more aggressive variants of MCL (blastic, pleomorphic, TP53+, or Ki67 > 60\%) showed poor outcome (Table 2), also after upfront ASCT according to the MCL2 protocol (Fig. $3 \mathrm{C}$ ). CR compared with PR, regardless whether obtained from PET/CT or CT, was highly predictive of outcome (OS, $\mathrm{P}=0.039 ; \mathrm{LSS}, \mathrm{P}=0.044 ; \mathrm{PFS}=0.025 ; \mathrm{Fig}$. 3D). Late relapses were seen.

\section{T-cell lymphomas}

78 out of 85 patients had peripheral T-cell lymphoma, and 56/78 were transplanted upfront in or according to the Nordic NLG-T-01 trial [20]; these patients showed good outcome (Fig. 3E) with 5-year OS at 75\% (Table 2). The two-year OS/PFS in patients with peripheral T-cell lymphoma were $91 \% / 78 \%$ if PET/CTCR, 33\%/33\% if PET/CT-PR ( $\mathrm{P}=0.024$; Table 3$)$. In contrast, CT could not predict outcome.

\section{Classical Hodgkin lymphoma}

These 60 patients, all relapsed/refractory, showed excellent outcome (OS at 5 years, 83\%; Table 2). However, refractory disease portended poorer survival than relapsed disease (Fig. 3F; OS, P = 0.002; LSS, P = 0.022; PFS, P = 0.021). In PET/CT-CR, 2-year OS/PFS were 100\%/89\% and in PET/CT-PR 75\%/50\%, but there were too few events to achieve statistical significance (Table 3).

\section{Relapse after ASCT}

The estimated fraction of relapses at 2 years were with PET/CT-CR 18\%, PET/CT-PR 41\%, CT-CR 29\%, CT-PR $43 \%$. 205 out of 521 patients relapsed after ASCT, at a median time of 308 days. Of these 205 patients 118 died from lymphoma, 3 from treatment toxicity, 18 from other causes, while 66 stayed alive. Relapse was most common in indolent (53\%), transformed (42\%), and primary aggressive (44\%) B-cell lymphoma. It was seen in $36 \%, 35 \%$, and $25 \%$ of mantle cell, Tcell, and Hodgkin lymphoma. Five-year OS after relapse was 30\%, and particularly poor in aggressive B-cell lymphoma (16\%), but $51 \%$ in Hodgkin lymphoma. The 44 patients who underwent allogeneic transplantation for relapsed disease showed better survival than those who did not (5-year OS $63 \%$ vs $21 \%$; $P<$ 0.00005), particularly in aggressive or transformed B-cell lymphoma (5-year OS 70\% vs $11 \%$; $P<0.00005)$. No patient had undergone CAR-T cell therapy at last follow-up. Long-term adverse events in our patients, including myeloid disease, have been described previously [2].

\section{Discussion}

This population-based single-center study shows that outcome after ASCT has improved with increasing PET/CT use. PET/CT may verify metabolic remission, identifying patients with truly chemosensitive disease, who have excellent outcome with ASCT. Hence, BEAM followed by ASCT thus remains an effective treatment option in lymphoma.

2-year OS and PFS in relapsed/refractory primary aggressive B-cell lymphoma were $58 \%$ and $50 \%$; the corresponding numbers for relapsed/refractory transformed B-cell lymphomas were $64 \%$ and $55 \%$. These figures are not inferior to those reported from the large CAR-T cell trials. In the axicabtagene ciloleucel trial, PFS was $42 \%$ at a median follow-up of 15.4 months [21]. In the tisagenlecleucel trial, the intention-to-treat analysis showed OS to be $40 \%$ at 1 year, and, in the patients who did proceed to receive CAR-T cell therapy, PFS was about 35\% at 18 months [22]. Lastly, the lisocabtagene maraleucel trial showed a 1-year PFS of 44\% [23]. Granted, these two modalities are not directly comparable, because only responders to chemotherapy proceed to ASCT and the follow-up times differ; on the other hand, many patients were excluded from therapy in the CAR-T cell trials because of disease progression between leukapheresis and CAR-T cell infusion, and the numbers come from clinical trials, not real-world practice, although emerging real-world CAR-T data appear to be equally good [24]. Furthermore, CAR-T cells are vastly more useful than ASCT for patients with stable disease after salvage induction therapy, and, of course, for those who relapse after ASCT. However, with PET/CT, one may identify those patients with aggressive B-cell lymphomas who will benefit from ASCT consolidation: in relapsed/refractory patients with PET/CT-CR, 2-year OS/PFS was 87\%/75\%. This excellent outcome for PET-negative relapsed/refractory patients is not inferior to the outcome of patients who attained complete remission in the CAR-T cell trials (PFS between $65 \%$ and $85 \%$ at 1 year) [21-23]-again the comparison limps somewhat because of differences in remission status and patient selection (particularly chemosensitivity) prior to the respective procedure. In our material, it also appears that the small proportion of patients who only have one site of PET positivity might be cured by with ASCT followed by local irradiation or surgery conducted after the bone-marrow regeneration. Involved-field radiotherapy to PET-positive lesions prior to ASCT has been shown to be a successful approach [25]. In patients with peripheral T-cell lymphoma transplanted upfront, 2-year OS/PFS after ASCT was 
OS/PFS $89 \% / 74 \%$. These figures compare favourably with other series, including one in which some of our patients participated [20, 26, 27]. Outcome in PET/CT-CR patients with Hodgkin lymphoma was excellent: 2-year OS/PFS 100\%/89\%.

In mantle cell lymphoma and indolent lymphoma, OS and PFS after ASCT were similar to previous reports, and ASCT remains part of standard therapy [19, 28]. However, late relapses do occur after ASCT; the 10-year PFS was in follicular lymphoma $31 \%$ and in mantle cell lymphoma $43 \%$. We believe that CAR-T cells $[29,30]$ and bispecific antibodies [31, 32] might improve long-term outcome for these slow-growing entities.

In our patients, survival after ASCT improved from 2015, without any improvement of NRM, thus this is probably due to a better identification of patients who should and should not proceed to ASCT. Karolinska's standard today is upfront ASCT in first remission for patients with transformed indolent B-cell, mantle cell, and peripheral T-cell lymphoma (except allogeneic SCT for hepatosplenic lymphoma), and ASCT in remitting relapsed/refractory aggressive B-cell lymphoma and (if first-line immunochemotherapy was $<2$ years ago) for relapsed/refractory follicular and nodal marginal zone lymphoma. A PET/CT is done before every ASCT. Patients who have attained PET/CT-CR proceed to ASCT, having a good chance for cure in aggressive lymphoma (2-year PFS:

relapsed/refractory aggressive B-cell lymphoma 75\%, relapsed/refractory classical Hodgkin lymphoma 89\%, upfront peripheral T-cell lymphoma $74 \%$ ). In the small number of patients with PET/CT-PR-local (a solitary active tumour), patients are given local irradiation after ASCT, with an acceptable cure rate (2-year PFS, 60\%). Patients who do not attain PET/CT-CR or PET/CT-PR-local are considered for treatment with CAR-T cells or trials with bispecific antibodies, or, if not available, experimental salvage regimens to induce better remission status and then ASCT.

With ASCT, the real treatment for lymphoma is the preceding conditioning regimen. Several conditioning regimens exist. Between 2015 and 2019 , we used BEAC as an alternative to BEAM because of melphalan shortages. The patients treated with BEAC showed inferior LSS, compared with those who received BEAM. It should be noted that Karolinska's BEAM regimen, used throughout this 25 -year period, has its own cytarabine schedule, with daily $400 \mathrm{mg} / \mathrm{m}^{2} 12-$ hour infusions, instead of the more common [33] two daily $200 \mathrm{mg} / \mathrm{m}^{2}$ 1-hour infusions. Infusion times correlate with cytarabine efficacy and toxicity, since the exposure to ara-C triphosphate (the active metabolite) is increased with longer infusions (cytarabine disappears from the plasma with a half-life of 7-13 minutes) $[34,35]$.

We conclude that an increasingly PET/CT-based selection of patients prior to ASCT has improved outcome after ASCT for lymphoma. For patients with PET/CT-CR, conditioning with BEAM followed by ASCT remains a strong standard treatment for several lymphoma entities. Further research is needed to identify the place of ASCT among other emerging salvage techniques.

\section{Abbreviations}

ASCT, autologous stem-cell transplantation

CAR, chimeric antigen receptor

$\mathrm{CR}$, complete response

$\mathrm{CT}$, computerized tomography

LSS, lymphoma-specific survival

NRM, non-relapse mortality

OS, overall survival

$\mathrm{PET} / \mathrm{CT}$, positron emission tomography/computerized tomography

PFS, progression-free survival

PR, partial response

TRM, treatment-related mortality

\section{Declarations}

Ethics approval. This study was approved by the Ethics Committee, Stockholm (Ref no. 2012/783-31/3 with Amendments 2015/327-32 and 2016/2379-32). Consent was waived.

Consent for publication. Not applicable.

Availability of data and material. The datasets during and/or analysed during the current study available from the corresponding author on reasonable request.

Competing interests. The authors declare that they have no competing interests.

Authors' contributions. BEW planned the study. $\mathrm{KN}, \mathrm{MC}$ and BEW collected data. BEW analyzed data. $\mathrm{KN}, \mathrm{MC}, \mathrm{KS}$, and BEW interpreted data. $\mathrm{KN}$, MC, KS, and BEW wrote the manuscript. 
Funding. This work was supported by Stockholm County Council (clinical research appointment), Cancerfonden, and Svenska Sällskapet för Medicinsk Forskning (SSMF).

Acknowledgments. The authors thank all patients, colleagues, and coworkers at the Haematology Unit, Karolinska.

\section{References}

1. Kuruvilla J. The role of autologous and allogeneic stem cell transplantation in the management of indolent B-cell lymphoma. Blood 2016; 127: 20932100.

2. Carlsten $M$, Jadersten $M$, Hellstrom A et al. The Karolinska experience of autologous stem-cell transplantation for lymphoma: a population-based study of all 433 patients 1994-2016. Exp Hematol Oncol 2019; 8: 7.

3. Cheson BD, Fisher RI, Barrington SF et al. Recommendations for initial evaluation, staging, and response assessment of Hodgkin and non-Hodgkin lymphoma: the Lugano classification. J Clin Oncol 2014; 32: 3059-3068.

4. Spaepen K, Stroobants S, Dupont P et al. Prognostic value of pretransplantation positron emission tomography using fluorine 18-fluorodeoxyglucose in patients with aggressive lymphoma treated with high-dose chemotherapy and stem cell transplantation. Blood 2003; 102: 53-59.

5. Schot B, van Imhoff G, Pruim J et al. Predictive value of early 18F-fluoro-deoxyglucose positron emission tomography in chemosensitive relapsed lymphoma. Br J Haematol 2003; 123: 282-287.

6. Alousi AM, Saliba RM, Okoroji GJ et al. Disease staging with positron emission tomography or gallium scanning and use of rituximab predict outcome for patients with diffuse large B-cell lymphoma treated with autologous stem cell transplantation. Br J Haematol 2008; 142: 786-792.

7. Schot BW, Zijlstra JM, Sluiter WJ et al. Early FDG-PET assessment in combination with clinical risk scores determines prognosis in recurring lymphoma. Blood 2007; 109: 486-491.

8. Derenzini E, Musuraca G, Fanti S et al. Pretransplantation positron emission tomography scan is the main predictor of autologous stem cell transplantation outcome in aggressive B-cell non-Hodgkin lymphoma. Cancer 2008; 113: 2496-2503.

9. Dickinson M, Hoyt R, Roberts AW et al. Improved survival for relapsed diffuse large B cell lymphoma is predicted by a negative pre-transplant FDG-PET scan following salvage chemotherapy. Br J Haematol 2010; 150: 39-45.

10. Devillier R, Coso D, Castagna $L$ et al. Positron emission tomography response at the time of autologous stem cell transplantation predicts outcome of patients with relapsed and/or refractory Hodgkin's lymphoma responding to prior salvage therapy. Haematologica 2012; 97: 1073-1079.

11. Roland V, Bodet-Milin C, Moreau A et al. Impact of high-dose chemotherapy followed by auto-SCT for positive interim [18F] FDG-PET diffuse large B-cell lymphoma patients. Bone Marrow Transplant 2011; 46: 393-399.

12. Moskowitz $\mathrm{CH}$, Matasar MJ, Zelenetz AD et al. Normalization of pre-ASCT, FDG-PET imaging with second-line, non-cross-resistant, chemotherapy programs improves event-free survival in patients with Hodgkin lymphoma. Blood 2012; 119: 1665-1670.

13. Armand P, Welch S, Kim HT et al. Prognostic factors for patients with diffuse large B cell lymphoma and transformed indolent lymphoma undergoing autologous stem cell transplantation in the positron emission tomography era. Br J Haematol 2013; 160: 608-617.

14. Cohen JB, Hall NC, Ruppert AS et al. Association of pre-transplantation positron emission tomography/computed tomography and outcome in mantle cell lymphoma. Bone Marrow Transplant 2013; 48: 1212-1217.

15. Magnusson E, Cao Q, Linden MA et al. Hematopoietic cell transplantation for mantle cell lymphoma: predictive value of pretransplant positron emission tomography/computed tomography and bone marrow evaluations for outcomes. Clin Lymphoma Myeloma Leuk 2014; 14: 114-121.

16. Bailly C, Carlier T, Berriolo-Riedinger A et al. Prognostic value of FDG-PET in patients with mantle cell lymphoma: results from the LyMa-PET Project. Haematologica 2020; 105: e33-e36.

17. Ahn SY, Jung SY, Jung SH et al. Prognostic significance of FDG-PET/CT in determining upfront autologous stem cell transplantation for the treatment of peripheral T cell lymphomas. Ann Hematol 2020; 99: 83-91.

18. Kedmi M, Avivi I, Ribakovsky E et al. Is there a role for therapy response assessment with 2-[fluorine-18] fluoro-2-deoxy-D-glucose-positron emission tomography/computed tomography in mantle cell lymphoma? Leuk Lymphoma 2014; 55: 2484-2489.

19. Geisler $\mathrm{CH}$, Kolstad A, Laurell A et al. Long-term progression-free survival of mantle cell lymphoma after intensive front-line immunochemotherapy with in vivo-purged stem cell rescue: a nonrandomized phase 2 multicenter study by the Nordic Lymphoma Group. Blood 2008; 112: $2687-2693$.

20. d'Amore F, Relander T, Lauritzsen GF et al. Up-front autologous stem-cell transplantation in peripheral T-cell lymphoma: NLG-T-01. J Clin Oncol 2012; 30: 3093-3099.

21. Neelapu SS, Locke FL, Bartlett NL et al. Axicabtagene Ciloleucel CAR T-Cell Therapy in Refractory Large B-Cell Lymphoma. N Engl J Med 2017; 377: 25312544.

22. Schuster SJ, Bishop MR, Tam CS et al. Tisagenlecleucel in Adult Relapsed or Refractory Diffuse Large B-Cell Lymphoma. N Engl J Med 2019; $380: 45-56$.

23. Abramson JS, Palomba ML, Gordon LI et al. Lisocabtagene maraleucel for patients with relapsed or refractory large B-cell lymphomas (TRANSCEND NHL 001): a multicentre seamless design study. Lancet 2020; 396: 839-852.

24. Vercellino L, Di Blasi R, Kanoun S et al. Predictive factors of early progression after CAR T-cell therapy in relapsed/refractory diffuse large B-cell lymphoma. Blood Adv 2020; 4: 5607-5615.

25. Hoppe BS, Moskowitz $\mathrm{CH}$, Zhang Z et al. The role of FDG-PET imaging and involved field radiotherapy in relapsed or refractory diffuse large B-cell lymphoma. Bone Marrow Transplant 2009; 43: 941-948. 
26. Reimer P, Rüdiger T, Geissinger E et al. Autologous stem-cell transplantation as first-line therapy in peripheral T-cell lymphomas: results of a prospective multicenter study. J Clin Oncol 2009; 27: 106-113.

27. Corradini P, Vitolo U, Rambaldi A et al. Intensified chemo-immunotherapy with or without stem cell transplantation in newly diagnosed patients with peripheral T-cell lymphoma. Leukemia 2014; 28: 1885-1891.

28. Jimenez-Ubieto A, Grande C, Caballero D et al. Autologous Stem Cell Transplantation for Follicular Lymphoma: Favorable Long-Term Survival Irrespective of Pretransplantation Rituximab Exposure. Biol Blood Marrow Transplant 2017; 23: 1631-1640.

29. Wang M, Munoz J, Goy A et al. KTE-X19 CAR T-Cell Therapy in Relapsed or Refractory Mantle-Cell Lymphoma. N Engl J Med 2020; 382: 1331-1342.

30. Hirayama AV, Gauthier J, Hay KA et al. High rate of durable complete remission in follicular lymphoma after CD19 CAR-T cell immunotherapy. Blood 2019; 134: 636-640.

31. Schuster SJ, Bartlett NL, Assouline S et al. Mosunetuzumab Induces Complete Remissions in Poor Prognosis Non-Hodgkin Lymphoma Patients, Including Those Who Are Resistant to or Relapsing After Chimeric Antigen Receptor T-Cell (CAR-T) Therapies, and Is Active in Treatment through Multiple Lines. Blood 2019; 134: 6-6.

32. Topp MS, Arnason J, Advani R et al. CLINICAL ACTIVITY OF REGN1979, AN ANTI-CD20 X ANTI-CD3 BISPECIFIC ANTIBODY (AB) IN PATIENTS (PTS) WITH (W/) RELAPSED/REFRACTORY (R/R) B-CELL NON-HODGKIN LYMPHOMA (B-NHL). Hematological Oncology 2019; 37 : 90-92.

33. Ferme C, Mounier N, Divine M et al. Intensive salvage therapy with high-dose chemotherapy for patients with advanced Hodgkin's disease in relapse or failure after initial chemotherapy: results of the Groupe d'Etudes des Lymphomes de l'Adulte H89 Trial. J Clin Oncol 2002; 20: 467-475.

34. Breithaupt H, Pralle H, Eckhardt T et al. Clinical results and pharmacokinetics of high-dose cytosine arabinoside (HD ARA-C). Cancer $1982 ; 50: 1248-1257$. 35. Stentoft J. The toxicity of cytarabine. Drug Saf 1990; 5: 7-27.

\section{Tables}




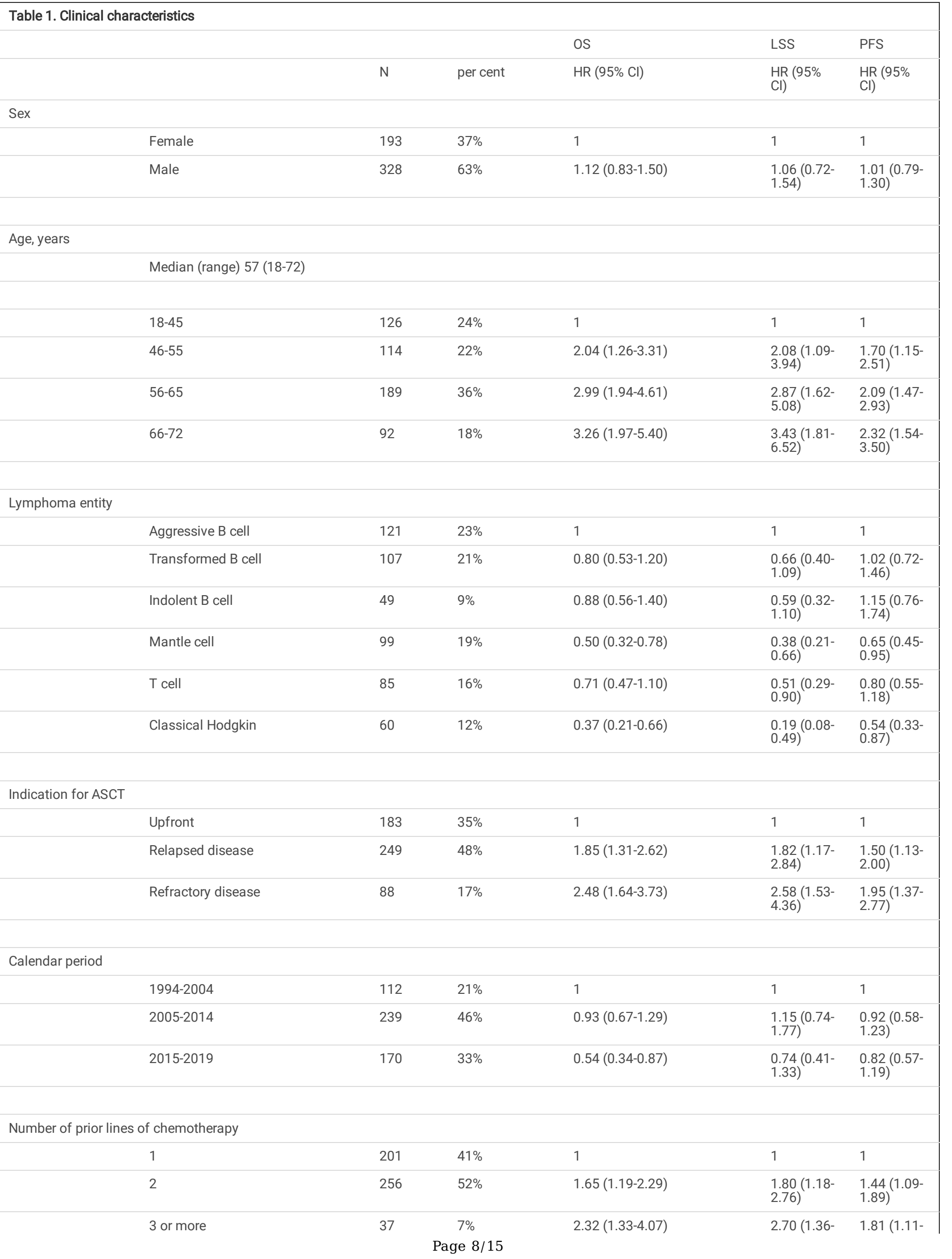


Conditioning regimen

\begin{tabular}{|c|c|c|c|c|c|}
\hline BEAM & 497 & $95 \%$ & 1 & 1 & 1 \\
\hline BEAC & 20 & $4 \%$ & $1.22(0.57-2.61)$ & $\begin{array}{l}1.57(0.69- \\
3.59)\end{array}$ & $\begin{array}{l}1.59(0.88- \\
2.84)\end{array}$ \\
\hline BCNU-Thiotepa & 3 & $1 \%$ & NA & NA & NA \\
\hline Су-ТВI & 1 & $0 \%$ & NA & NA & NA \\
\hline
\end{tabular}

Rituximab prior to ASCT in B-cell disease

\begin{tabular}{|c|c|c|c|c|c|}
\hline No & 63 & $17 \%$ & 1 & 1 & 1 \\
\hline Yes & 313 & $83 \%$ & $0.61(0.42-0.87)$ & $\begin{array}{l}0.76(0.47- \\
1.22)\end{array}$ & $\begin{array}{l}0.73(0.53- \\
1.02)\end{array}$ \\
\hline
\end{tabular}

Abbreviations: OS, overall survival; LSS, lymphoma-specific survival, PFS, progression-free survival; ASCT, autologous stemcell transplantation; NA, not analyzable 


\begin{tabular}{|c|c|c|c|c|c|c|c|c|c|c|c|c|c|}
\hline & & OS & & & & LSS & & & & PFS & & & \\
\hline & $\mathrm{N}$ & $2 y$ & $5 y$ & $10 y$ & $P$ & $2 y$ & $5 y$ & $10 y$ & $P$ & $2 y$ & $5 y$ & $10 y$ & $P$ \\
\hline All lymphomas & 521 & $74 \%$ & $66 \%$ & $56 \%$ & & $82 \%$ & $76 \%$ & $70 \%$ & & $64 \%$ & $52 \%$ & $42 \%$ & \\
\hline Primary aggressive B-cell lymphomas & 121 & $59 \%$ & $53 \%$ & $47 \%$ & & $67 \%$ & $64 \%$ & $56 \%$ & & $52 \%$ & $45 \%$ & $41 \%$ & \\
\hline DLBCL or high-grade B-cell lymphoma & 105 & $59 \%$ & $52 \%$ & $44 \%$ & & $68 \%$ & $64 \%$ & $55 \%$ & & $50 \%$ & $42 \%$ & $38 \%$ & \\
\hline Not-DLBCL or high-grade B-cell lymphoma & 16 & $60 \%$ & $60 \%$ & $60 \%$ & 0.28 & $64 \%$ & $64 \%$ & $64 \%$ & 0.55 & $61 \%$ & $61 \%$ & $61 \%$ & 0.14 \\
\hline FL grade 3B & 2 & & & & & & & & & & & & \\
\hline Burkitt lymphoma & 3 & & & & & & & & & & & & \\
\hline PTLD & 2 & & & & & & & & & & & & \\
\hline PMBCL or greyzone lymphoma & 3 & & & & & & & & & & & & \\
\hline Plasmablastic lymphoma & 5 & & & & & & & & & & & & \\
\hline $\begin{array}{l}\text { Lymphomatoid granulomatosis grade } \\
\text { III }\end{array}$ & 1 & & & & & & & & & & & & \\
\hline Upfront patients & 7 & $86 \%$ & $86 \%$ & $86 \%$ & & $100 \%$ & $100 \%$ & $100 \%$ & & $86 \%$ & $86 \%$ & $86 \%$ & \\
\hline Relapsed/refractory patients & 114 & $58 \%$ & $51 \%$ & $44 \%$ & 0.19 & $65 \%$ & $62 \%$ & $53 \%$ & 0.070 & $50 \%$ & $43 \%$ & $38 \%$ & 0.09 \\
\hline Transformed B-cell lymphomas & 107 & $71 \%$ & $63 \%$ & $52 \%$ & & $78 \%$ & $69 \%$ & $64 \%$ & & $59 \%$ & $42 \%$ & $35 \%$ & \\
\hline Transformed FL & 72 & $76 \%$ & $71 \%$ & $56 \%$ & & $81 \%$ & $75 \%$ & $68 \%$ & & $65 \%$ & $48 \%$ & $38 \%$ & \\
\hline Transformed not-FL & 35 & $61 \%$ & $41 \%$ & $41 \%$ & 0.22 & $73 \%$ & $49 \%$ & $49 \%$ & 0.35 & $48 \%$ & $30 \%$ & $30 \%$ & 0.28 \\
\hline Transformed MZL & 18 & & & & & & & & & & & & \\
\hline Transformed CLL/SLL (Richter) & 5 & & & & & & & & & & & & \\
\hline Transformed WM & 3 & & & & & & & & & & & & \\
\hline Transformed NLPHL & 2 & & & & & & & & & & & & \\
\hline Transformed indolent B-cell lymphoma & 7 & & & & & & & & & & & & \\
\hline Upfront patients & 28 & $92 \%$ & $92 \%$ & $92 \%$ & & $68 \%$ & $55 \%$ & $41 \%$ & & $95 \%$ & $95 \%$ & $95 \%$ & \\
\hline Relapsed/refractory patients & 79 & $64 \%$ & $54 \%$ & $41 \%$ & 0.006 & $73 \%$ & $61 \%$ & $55 \%$ & 0.009 & $55 \%$ & $38 \%$ & $33 \%$ & 0.13 \\
\hline $\begin{array}{l}\text { Primary and transformed aggressive B-cell } \\
\text { lymphomas }\end{array}$ & 228 & $64 \%$ & $58 \%$ & $50 \%$ & & $72 \%$ & $67 \%$ & $60 \%$ & & $55 \%$ & $44 \%$ & $39 \%$ & \\
\hline Primary aggressive & 121 & $59 \%$ & $53 \%$ & $47 \%$ & & $67 \%$ & $64 \%$ & $56 \%$ & & $52 \%$ & $45 \%$ & $41 \%$ & \\
\hline Transformed aggressive & 107 & $71 \%$ & $63 \%$ & $52 \%$ & 0.20 & $78 \%$ & $69 \%$ & $64 \%$ & 0.09 & $59 \%$ & $42 \%$ & $35 \%$ & 0.93 \\
\hline Indolent B-cell lymphomas & 49 & $80 \%$ & $59 \%$ & $44 \%$ & & $90 \%$ & $75 \%$ & $65 \%$ & & $60 \%$ & $45 \%$ & $26 \%$ & \\
\hline FL grade $1-3 A$ & 39 & $78 \%$ & $57 \%$ & $43 \%$ & & $88 \%$ & $76 \%$ & $69 \%$ & & $60 \%$ & $44 \%$ & $31 \%$ & \\
\hline Not-FL grade $1-3 \mathrm{~A}$ & 10 & $89 \%$ & $63 \%$ & $51 \%$ & 0.55 & $100 \%$ & $71 \%$ & $57 \%$ & 0.24 & $57 \%$ & $46 \%$ & $11 \%$ & 0.48 \\
\hline MZL & 1 & & & & & & & & & & & & \\
\hline CLL/SLL & 6 & & & & & & & & & & & & \\
\hline WM & 1 & & & & & & & & & & & & \\
\hline NLPHL & 2 & & & & & & & & & & & & \\
\hline All indolent lymphomas & 156 & $74 \%$ & $61 \%$ & $48 \%$ & & $82 \%$ & $71 \%$ & $64 \%$ & & $59 \%$ & $43 \%$ & $31 \%$ & \\
\hline Not transformed & 49 & $80 \%$ & $59 \%$ & $44 \%$ & & $90 \%$ & $75 \%$ & $65 \%$ & & $60 \%$ & $45 \%$ & $26 \%$ & \\
\hline
\end{tabular}




\begin{tabular}{|c|c|c|c|c|c|c|c|c|c|c|c|c|c|}
\hline Transformed & 107 & $71 \%$ & $63 \%$ & $52 \%$ & 0.77 & $78 \%$ & $69 \%$ & $64 \%$ & 0.65 & $59 \%$ & $42 \%$ & $35 \%$ & 0.77 \\
\hline All follicular lymphomas & 111 & $77 \%$ & $65 \%$ & $50 \%$ & & $83 \%$ & $75 \%$ & $68 \%$ & & $63 \%$ & $47 \%$ & $35 \%$ & \\
\hline Not transformed & 39 & $78 \%$ & $57 \%$ & $43 \%$ & & $88 \%$ & $76 \%$ & $69 \%$ & & $60 \%$ & $44 \%$ & $31 \%$ & \\
\hline Transformed & 72 & $76 \%$ & $71 \%$ & $56 \%$ & 0.58 & $81 \%$ & $75 \%$ & $68 \%$ & 0.63 & $65 \%$ & $48 \%$ & $38 \%$ & 0.69 \\
\hline Mantle cell lymphoma & 99 & $86 \%$ & $75 \%$ & $73 \%$ & & $91 \%$ & $82 \%$ & $82 \%$ & & $80 \%$ & $62 \%$ & $43 \%$ & \\
\hline Classical & 83 & $90 \%$ & $78 \%$ & $78 \%$ & & $95 \%$ & $86 \%$ & $86 \%$ & & $84 \%$ & $67 \%$ & $47 \%$ & \\
\hline Non-classical & 16 & $66 \%$ & $58 \%$ & $43 \%$ & 0.021 & $70 \%$ & $62 \%$ & $62 \%$ & 0.025 & $61 \%$ & $37 \%$ & $18 \%$ & 0.005 \\
\hline Blastic & 9 & & & & & & & & & & & & \\
\hline Pleomorphic or P53+ or Ki67>60\% & 7 & & & & & & & & & & & & \\
\hline Upfront patients & 87 & $90 \%$ & $78 \%$ & $78 \%$ & & $93 \%$ & $84 \%$ & $84 \%$ & & $83 \%$ & $66 \%$ & $45 \%$ & \\
\hline Relapsed/refractory patients & 12 & $58 \%$ & $49 \%$ & $32 \%$ & 0.006 & $81 \%$ & $67 \%$ & $67 \%$ & 0.30 & $58 \%$ & $40 \%$ & $40 \%$ & 0.054 \\
\hline T-cell lymphomas & 85 & $74 \%$ & $69 \%$ & $53 \%$ & & $83 \%$ & $79 \%$ & $71 \%$ & & $65 \%$ & $54 \%$ & $45 \%$ & \\
\hline PTCL & 78 & $79 \%$ & $73 \%$ & $56 \%$ & & $87 \%$ & $83 \%$ & $74 \%$ & & $70 \%$ & $58 \%$ & $48 \%$ & \\
\hline Non-PTCL & 7 & $18 \%$ & $18 \%$ & ND & 0.004 & $25 \%$ & $25 \%$ & ND & 0.013 & $0 \%$ & $0 \%$ & $0 \%$ & 0.002 \\
\hline $\begin{array}{l}\text { Sézary/T-lymphoblastic } \\
\text { lymphoma/ATLL }\end{array}$ & 3 & & & & & & & & & & & & \\
\hline ENKTCL & 4 & & & & & & & & & & & & \\
\hline PTCL, ALCL & 25 & $79 \%$ & $79 \%$ & $74 \%$ & & $91 \%$ & $91 \%$ & $91 \%$ & & $80 \%$ & $75 \%$ & $70 \%$ & \\
\hline PTCL, non-ALCL & 53 & $78 \%$ & $69 \%$ & $41 \%$ & 0.08 & $85 \%$ & $78 \%$ & $63 \%$ & 0.09 & $65 \%$ & $47 \%$ & $33 \%$ & 0.023 \\
\hline AITL/FHTCL & 29 & & & & & & & & & & & & \\
\hline PTCL-NOS & 17 & & & & & & & & & & & & \\
\hline EATCL/HSTCL/SPTCL/Lennert & 7 & & & & & & & & & & & & \\
\hline Upfront PTCL patients & 56 & $81 \%$ & $75 \%$ & $67 \%$ & & $86 \%$ & $80 \%$ & $71 \%$ & & $70 \%$ & $60 \%$ & $55 \%$ & \\
\hline Relapsed/refractory PTCL patients & 22 & $73 \%$ & $68 \%$ & $39 \%$ & 0.07 & $89 \%$ & $89 \%$ & $80 \%$ & 0.44 & $68 \%$ & $54 \%$ & $38 \%$ & 0.21 \\
\hline Classical Hodgkin lymphoma & 60 & $85 \%$ & $83 \%$ & $74 \%$ & & $92 \%$ & $90 \%$ & $90 \%$ & & $71 \%$ & $66 \%$ & $60 \%$ & \\
\hline \multicolumn{14}{|c|}{$\begin{array}{l}\text { Abbreviations: OS, overall survival; LSS, lymphoma-specific survival, PFS, progression-free survival DLBCL, diffuse large B-cell lymphoma; FL, follicular } \\
\text { lymphoma; PTLD, post-transplantation lymphoproliferative disorde; PMBCL, primary mediastinal B-cell lymphoma; MZL, marginal zone lymphoma; CLL, } \\
\text { chronic lymphocytic leukemia; SLL, small lymphocytic lymphoma; WM, Waldenström macroglobulinemia; NLPHL, nodular lymphocyte-predominant } \\
\text { Hodgkin lymphoma; PTCL, peripheral T-cell lymphoma; ALCL, anaplastic large cell lymphoma; AITL, angioimmunoblastic T-cell lymphoma; FHTCL, follicula } \\
\text { helper T-cell lymphoma; EATL, enteropathy-associated T-cell lymphoma; HSTCL, hepatosplenic T-cell lymphoma; SPTCL, subcutaneous panniculitis-like T- } \\
\text { cell lymphoma; ATLL, adult T-cell leukemia/lymphoma; ENKTCL, extranodal NK/T-cell lymphoma, nasal type }\end{array}$} \\
\hline
\end{tabular}




\begin{tabular}{|c|c|c|c|c|c|c|c|c|c|c|c|}
\hline & & & os & & & LSS & & & PFS & & \\
\hline & $\mathrm{N}$ & per cent & $2 y$ & $5 y$ & $\mathrm{P}$ & $2 y$ & $5 y$ & $\mathrm{P}$ & $2 y$ & $5 y$ & $\mathrm{P}$ \\
\hline $\mathrm{PET} / \mathrm{CT}$ & 136 & $100 \%$ & & & & & & & & & \\
\hline $\mathrm{PET} / \mathrm{CT}-\mathrm{CR}$ & 105 & $77 \%$ & $93 \%$ & $88 \%$ & & $94 \%$ & $92 \%$ & & $81 \%$ & $67 \%$ & \\
\hline PET/CT-PR & 31 & $23 \%$ & $55 \%$ & $55 \%$ & 0.0003 & $68 \%$ & $68 \%$ & 0.0009 & $45 \%$ & $41 \%$ & 0.004 \\
\hline PET/CT-PR-local & 10 & $7 \%$ & $80 \%$ & $80 \%$ & & $90 \%$ & $90 \%$ & & $60 \%$ & $50 \%$ & \\
\hline PET/CT-PR-general & 21 & $15 \%$ & $41 \%$ & $41 \%$ & $<0.00005$ & $56 \%$ & $56 \%$ & 0.0002 & $36 \%$ & $36 \%$ & 0.003 \\
\hline Aggressive B-cell lymphoma & 72 & & & & & & & & & & \\
\hline PET/CT-CR & 50 & $69 \%$ & $90 \%$ & $76 \%$ & & $90 \%$ & $85 \%$ & & $73 \%$ & $55 \%$ & \\
\hline PET/CT-PR & 22 & $31 \%$ & $50 \%$ & $50 \%$ & 0.004 & $59 \%$ & $59 \%$ & 0.006 & $45 \%$ & $39 \%$ & 0.032 \\
\hline Relapsed/refractory & 54 & & & & & & & & & & \\
\hline $\mathrm{PET} / \mathrm{CT}-\mathrm{CR}$ & 36 & $67 \%$ & $87 \%$ & $64 \%$ & & $87 \%$ & $80 \%$ & & $75 \%$ & $48 \%$ & \\
\hline PET/CT-PR & 18 & $33 \%$ & $43 \%$ & $43 \%$ & 0.013 & $50 \%$ & $50 \%$ & 0.008 & $44 \%$ & $44 \%$ & 0.15 \\
\hline Peripheral T-cell lymphoma & 18 & & & & & & & & & & \\
\hline $\mathrm{PET} / \mathrm{CT}-\mathrm{CR}$ & 15 & $83 \%$ & $91 \%$ & $91 \%$ & & $91 \%$ & $91 \%$ & & $78 \%$ & $62 \%$ & \\
\hline PET/CT-PR & 3 & $17 \%$ & $33 \%$ & NA & 0.024 & $50 \%$ & NA & 0.19 & $33 \%$ & NA & 0.27 \\
\hline Classical Hodgkin lymphoma & 25 & & & & & & & & & & \\
\hline PET/CT-CR & 21 & $84 \%$ & $100 \%$ & $100 \%$ & & $100 \%$ & $100 \%$ & & $89 \%$ & $67 \%$ & \\
\hline PET/CT-PR & 4 & $16 \%$ & $75 \%$ & $75 \%$ & 0.083 & $100 \%$ & $100 \%$ & 1 & $50 \%$ & $50 \%$ & 0.37 \\
\hline CT & 235 & & & & & & & & & & \\
\hline CT-CR & 117 & $50 \%$ & $76 \%$ & $67 \%$ & & $83 \%$ & $78 \%$ & & $66 \%$ & $55 \%$ & \\
\hline CT-PR & 118 & $50 \%$ & $62 \%$ & $51 \%$ & 0.035 & $70 \%$ & $62 \%$ & 0.022 & $51 \%$ & $36 \%$ & 0.004 \\
\hline Aggressive B-cell lymphoma & 86 & & & & & & & & & & \\
\hline CT-CR & 34 & $40 \%$ & $59 \%$ & $52 \%$ & & $69 \%$ & $69 \%$ & & $50 \%$ & $35 \%$ & \\
\hline CT-PR & 52 & $60 \%$ & $52 \%$ & $47 \%$ & 0.81 & $57 \%$ & $52 \%$ & 0.25 & $46 \%$ & $37 \%$ & 0.85 \\
\hline Relapsed/refractory & 77 & & & & & & & & & & \\
\hline CT-CR & 30 & $39 \%$ & $50 \%$ & $45 \%$ & & $64 \%$ & $64 \%$ & & $46 \%$ & $30 \%$ & \\
\hline CT-PR & 47 & $61 \%$ & $49 \%$ & $44 \%$ & 0.99 & $54 \%$ & $49 \%$ & 0.38 & $43 \%$ & $35 \%$ & 0.99 \\
\hline Peripheral T-cell lymphoma & 44 & & & & & & & & & & \\
\hline CT-CR & 27 & $61 \%$ & $74 \%$ & $63 \%$ & & $77 \%$ & $71 \%$ & & $67 \%$ & $56 \%$ & \\
\hline CT-PR & 17 & $39 \%$ & $76 \%$ & $76 \%$ & 0.93 & $92 \%$ & $92 \%$ & 0.22 & $65 \%$ & $44 \%$ & 0.25 \\
\hline Classical Hodgkin lymphoma & 13 & & & & & & & & & & \\
\hline CT-CR & 3 & $23 \%$ & $100 \%$ & $100 \%$ & & $100 \%$ & $100 \%$ & & $67 \%$ & $67 \%$ & \\
\hline
\end{tabular}


Abbreviations: $\mathrm{PET} / \mathrm{CT}$, positron emission tomography/computerized tomography; $\mathrm{CT}$, computerized tomography; CR, complete remission; $\mathrm{PR}$, partial remission; NA, not analyzable

\section{Figures}

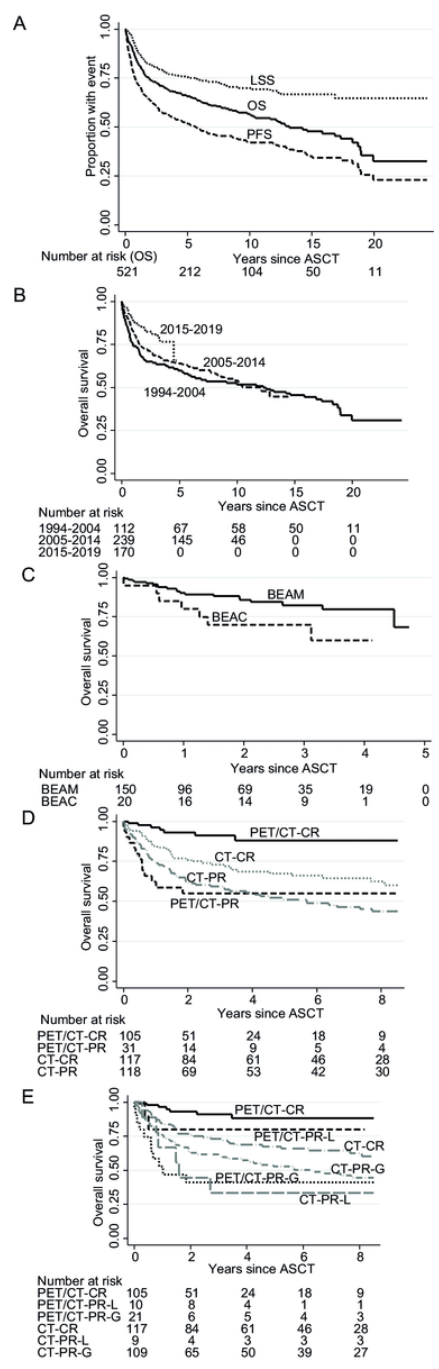

\section{Figure 1}

Kaplan-Meier graphs of (A) overall, lymphoma-specific, and progression-free survival in all patients (OS; LSS; PFS), (B) OS by calendar period, (C) BEAM or BEAC conditioning in the last calendar period, (D) partial or complete remission (PR; CR) status according to computerized tomography (CT) or 18flourodeoxyglucose positron emission tomography (PET)/CT, (E) PR or CR status according to CT and PET/CT with PR patients locally treated after ASCT (PRL) as a separate group, compared with generalized PR (PR-G); the last two graphs are cut at 8 years due to the different observation times between CT and PET/CT patients. Abbreviation: ASCT, autologous stem-cell transplantation. 
A
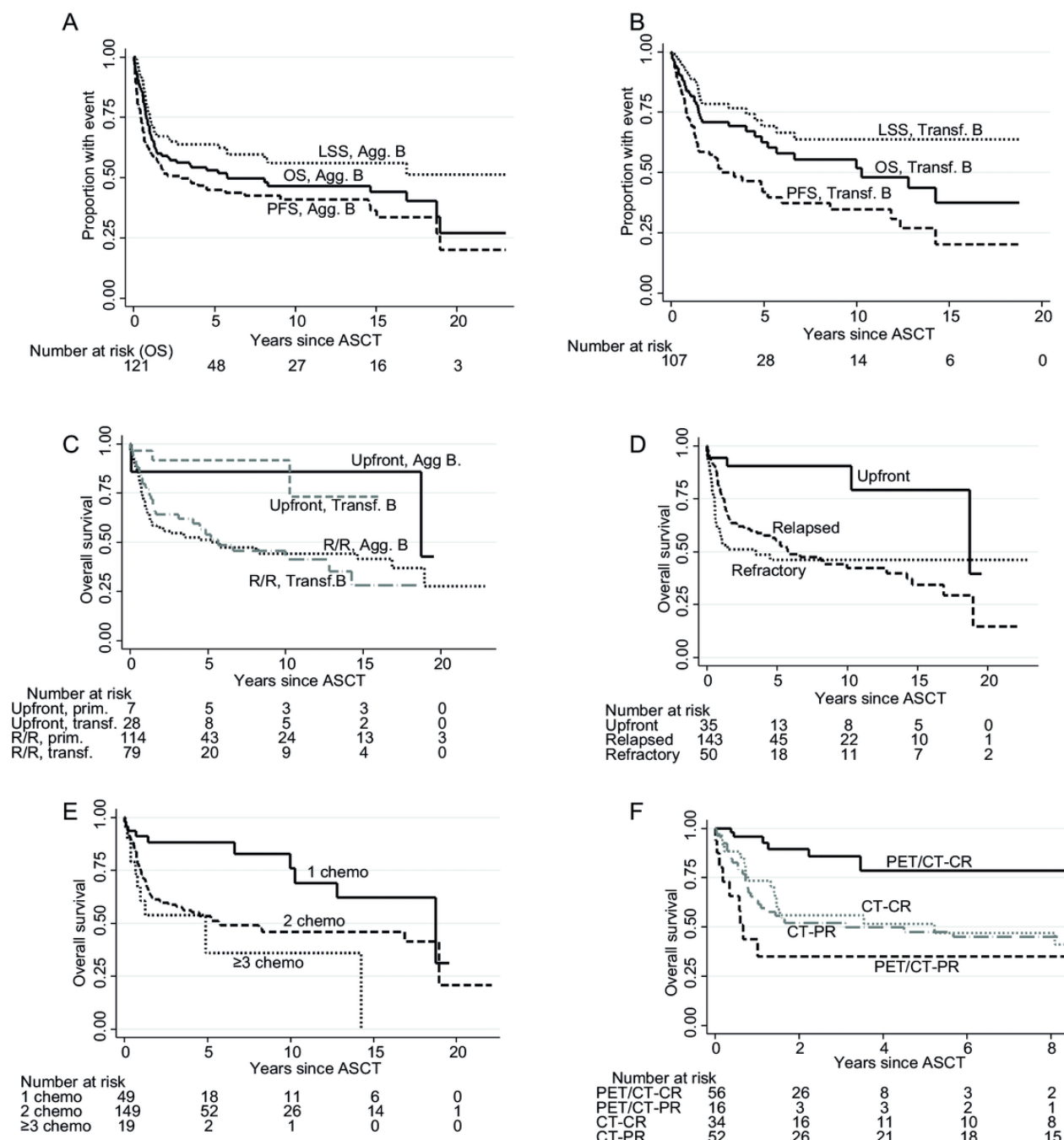
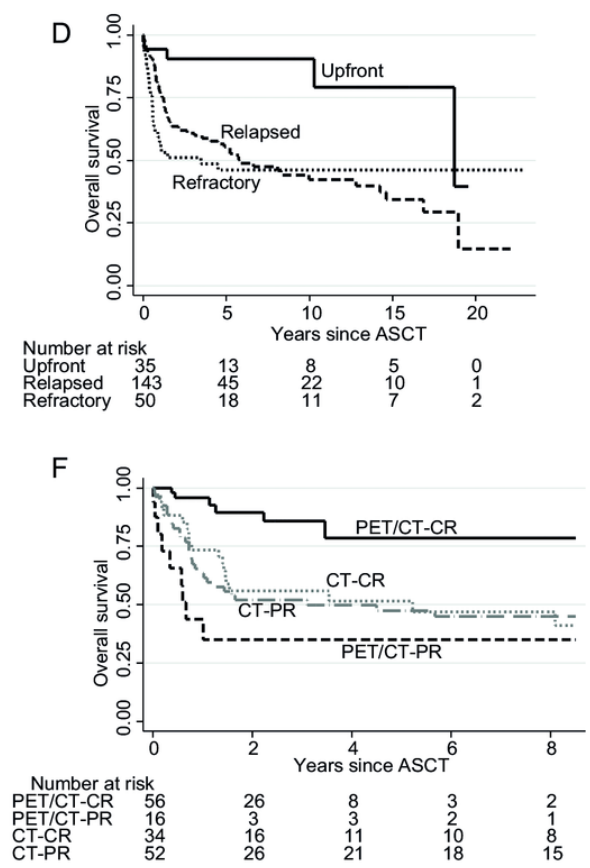

\section{Figure 2}

Kaplan-Meier graphs of overall, lymphoma-specific, and progression-free survival (OS; LSS; PFS) in (A) primary aggressive (Agg. B) and (B) transformed indolent (Transf. B) B-cell lymphoma, (C) OS by upfront and relapsed/refractory status in primary aggressive and transformed indolent B-cell lymphoma, (D) OS in primary aggressive or transformed indolent B-cell lymphoma patients by upfront, relapsed, or refractory status, (E) OS by number of preceding lines of chemotherapy, (F) OS by PR or CR status according to CT and PET/CT (with PET/CT-PR patients locally treated after ASCT grouped with PET/CT-CR); the last graph is cut at 8 years due to the different observation times between CT and PET/CT patients. Abbreviation: ASCT, autologous stem-cell transplantation. 

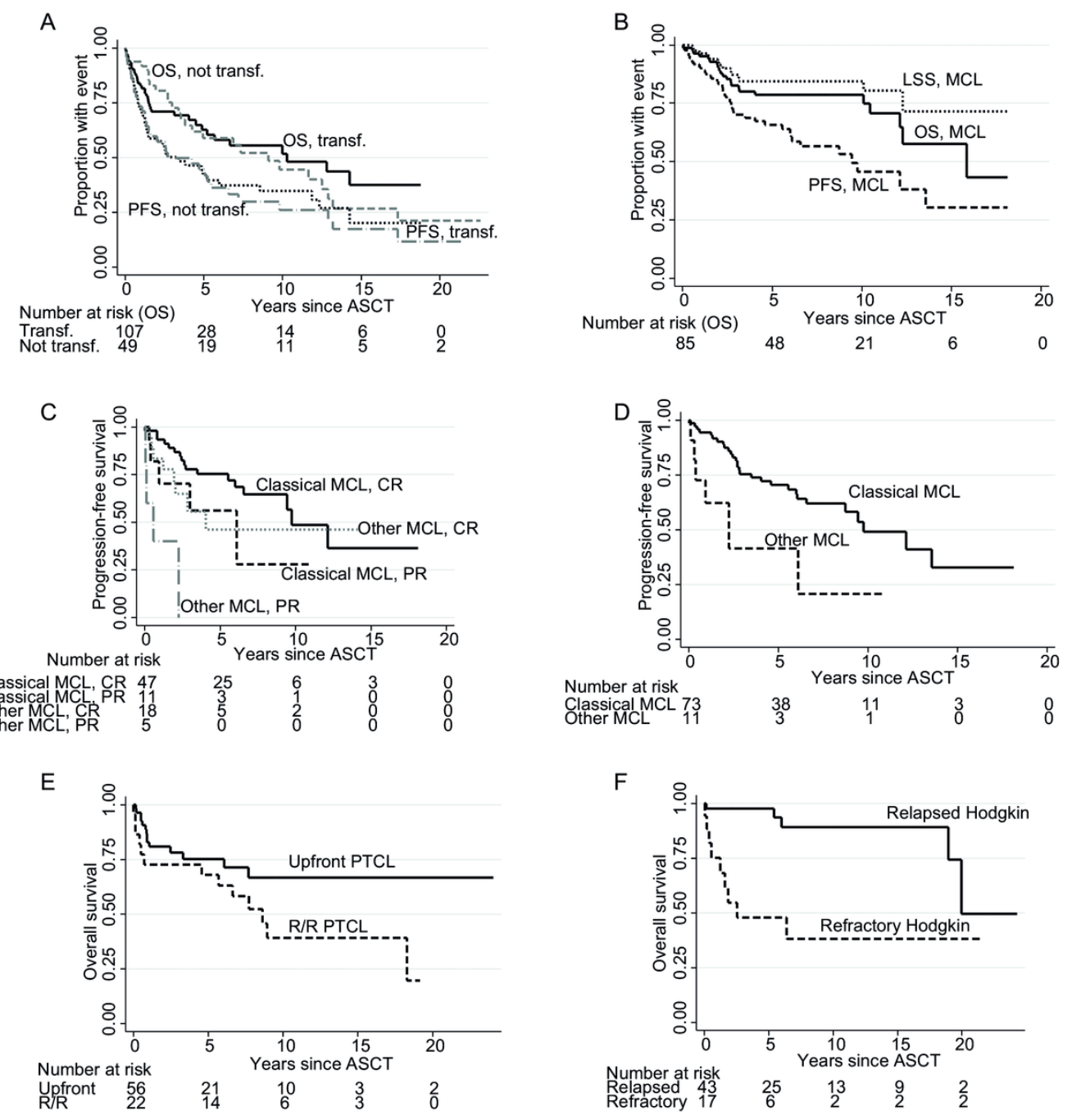

\section{Figure 3}

Kaplan-Meier graphs of (A) overall and progression-free survival (OS; PFS) in not-transformed (not transf.) and transformed indolent B-cell lymphoma, (B) OS, lymphoma-specific survival (LSS), and PFS in mantle cell lymphoma (MCL), (C) PFS by remission status in classical and other types of MCL, (D) PFS in classical and other types of MCL transplanted upfront, (E) OS in peripheral T-cell lymphoma by upfront or relapsed/refractory (R/R) status, (F) OS in Hodgkin lymphoma by relapsed or refractory status. Abbreviation: ASCT, autologous stem-cell transplantation. 